\title{
Dimensional Modeling of Indian Materials Database
}

\author{
Suja Ramachandran \\ Indira Gandhi Centre for Atomic \\ Research, Tamil Nadu, India
}

\author{
S. Rajeswari \\ Indira Gandhi Centre for Atomic \\ Research, Tamil Nadu, India
}

\author{
S.A.V. Satya Murty \\ Indira Gandhi Centre for Atomic \\ Research, Tamil Nadu, India
}

\begin{abstract}
The Indian Materials Database (IMDB) is a national project aiming to develop a database through compilation of materials property data available in different laboratories in India. The database contains data on mechanical, corrosion, nondestructive evaluation, thermal and optical properties of a wide variety of materials. Selecting the appropriate data modeling technique is crucial for the successful deployment of such a database. Dimensional modeling is a logical design technique to present data in a standard, intuitive framework that allows for high-performance access. Dimensional modeling of data results in a 'Star Schema', where the data constitutes a central fact table surrounded by dimension tables. This paper discusses the model and architecture of the material database using a 'Snowflake Schema', which is a variation of 'Star Schema', where some of the dimensions are normalized into multiple related tables. The database contains a central fact table linked to multiple dimensions namely, 1) Materials 2) Properties of materials 3) Details of experiments conducted on materials and 4) Source from which data is obtained.
\end{abstract}

\section{Keywords}

Database Models, Material Database, Data warehousing, Dimensional Design, Snowflake Schema.

\section{INTRODUCTION}

The Indian Materials Database (IMDB) is a national project for the development of a material database through compilation of materials property data available in different laboratories in India [1]. This project is a pioneer attempt to bring together the work of eminent metallurgists working in distinguished Indian organizations and make it available to the scientific community internationally. A unique feature of this data store will be the authenticity of data contained in it, since it solely consists of data published in reputed, peer-reviewed publications. Currently, data is available on Mechanical, Corrosion, Nondestructive Evaluation, Thermal and Optical properties of different materials used in power, space, chemical and infrastructural industries.

The concept of a comprehensive data store for materials arises from the need for a reliable, consolidated, unique and integrated storage for the data that originates from various research groups across the country. The amount of scientific data on materials generated in laboratories is growing rapidly, thus making the efficient storage and querying of this data a major challenge for the organizations. As time passes, this wealth of data will become an obstacle rather than an asset for scientific learning. Some of the information may appear in scientific publications in heterogeneous structures and proprietary formats, but, only a small portion will be stored in systems that are generally accessible.

In this project, the huge collection of diverse data from different research groups has eventually resulted in the creation of a warehouse of materials data. According to the definition given by Bill Inmon [2], "A data warehouse is a subject-oriented, integrated, time-variant and non-volatile collection of data in support of management's decision making process". In other words, data warehouse is the term used for a computer database which is the collection and storage of data from multiple and usually varied sources into one comprehensive and easily manipulated database. This collection is then used to manage information efficiently, analyze the collected data and perform data mining for deducing new information.

Selecting an appropriate data modeling technique forms the foundation for the successful deployment of such a data warehousing system, since it describes how data is to be represented and accessed. Dimensional modeling (DM) is a logical design technique often used for data warehouses ([3], [4], [5]). It seeks to present the data in a standard, intuitive framework that allows for high-performance access. Data warehouses are targeted for aiding decision support. The dimensional modeling aims to implement a database that eases user navigation and enhances decision support query performance. In a dimensional database, rather than representing data in multiple relations, key data entities are represented as different dimensions. In this way, it offers an extension to the relational database systems providing a multidimensional view of the data.

The rest of this paper is organized as follows: Section 2 describes the related works on the subject. Section 3 details the characteristic features of Indian Materials Database. The dimensional modeling process by which the database is modeled is presented in section 4 , and section 5 discusses the results of this work.

\section{RELATED WORKS}

The advantages and issues in using dimensional models for designing data warehouses have been extensively addressed in literature. Kimball et al. ([3], [4], [5]) provide a detailed discussion on designing data warehouses according to dimensional modeling concepts. The strengths of dimensional model over relational models in designing decision support systems are stated resolutely. A method for deriving dimensional schemas from traditional entity relationship schemas is presented in [6]. An E-R model is converted to a dimensional model by classifying entities, identifying hierarchies and aggregating transaction data.

A graphical conceptual model for data warehouses based on dimensional model called Dimensional Fact Model (DFM) is proposed in [7]. The model consists of a set of fact schemas whose basic elements are facts, measures, attributes, dimensions and hierarchies. A semi-automated methodology to build this Dimensional Fact Model from the pre-existing Entity-Relationship schemes describing a database is discussed in [8]. 
Song et al. [9] analyze many-to-many relationships that exist between fact and dimension tables in a dimensional model. Song and Jones [10] present Dimensional Design Patterns (DDPs) and their applications in the design of dimensional models. The DDPs assist data warehousing practitioners by providing an approach for identifying dimensions in a systematic and usable way. Martyn [11] details the two specialized logical schemas, star and snowflake, used in multi dimensional designs. The paper discusses the criteria to determine the best logical schema based on correctness, efficiency and usability. Levene and G. Loizou [12] formalize the concept of a snowflake schema in terms of an acyclic database schema and define a normal form for snowflake schemas.

Before starting the design process, different material databases and their design aspects were carefully studied. Xiu-Juan Zhang et al. [13] proposes a material database which covers homogeneous materials and different types of heterogeneous materials along with their properties such as mechanical, electrical, magnetic and thermodynamics. P.S. Ramalhete et al. [14] does a critical analysis of different material databases and digital tools available for material selection. The paper says that, in general databases, the methods and the properties used for selection are the name of material, family, class and subclass of materials and material properties. The European Fusion Material Properties Database [15] is a relational database developed to collect, expand and preserve the data produced on material properties and their critical parameters for fusion environments. The database contains data on raw experimental data points, data qualified by expert groups and processed material property data.

\section{THE INDIAN MATERIALS DATABASE}

As said earlier, the Indian Materials Database (IMDB) project is an opening step in accumulating the data on materials and their properties which originate from experiments conducted in various laboratories across the country. By bringing together the data on various properties of a number of materials along with graphs and microstructures on a single platform, this project helps scientists, metallurgists and engineers to compare and select materials needed for their specific applications.

Different properties of several materials are brought together in the Indian Material Database. Apart from the presence of materials and material properties, the data available is of heterogeneous nature, which has to be structured and presented in the database. Currently, the materials whose data are available in the data warehouse include:

- $\quad$ Steels - Austenitic Stainless Steel (Type 304, 306 and so on), Ferritic Steel (2.25Cr-1Mo, 9Cr-1Mo and so on), Duplex Stainless Steel, Carbon Steel, Mild Steel etc...

- Coatings and Thin films - Hard Coatings, Nano Composite Coatings, Nano Composite Thin Films etc...

- Metal Matrix Composites

- $\quad$ Nickel, Tin, Aluminum, Zirconium and Titanium Alloys

- Ductile Iron

Also, data regarding the following material properties are included in the material data warehouse:

- Mechanical Properties - Low Cycle Fatigue, Thermomechanical Fatigue, Tensile, Hardness, Creep, Fatigue Crack Growth, Dynamic Fracture Toughness, Creep Crack Growth etc....
- Corrosion Properties - Sensitization, Hydrogen Transport, Pitting, Sodium Corrosion, Biofouling, High Temperature Corrosion etc...

- Non Destructive Evaluation - Results of Eddy Current Testing, Ultrasonic Spectroscopy, Magnetic Flux Leakage

- Optical and Thermal Properties

The data on a material property can be in the form of tables, graphs, microstructure images or a combination of these. While representing data in tabular format, the test results obtained for each property contain diverse fields which differ from each other. For example, the Sensitization property contains data in the form "Temperature vs. Time to Sensitization". Similarly, the Hardness property data is of the form "Aging Time vs. Hardness". Data on some other properties are represented in more than one table. For example, the data on biofouling property constitute tables for frequency of occurrence of Manganese oxidizing bacterial species in the bio-film on titanium exposed to chlorinated sea water, Manganese and iron content in the bio-film formed on titanium surface exposed to chlorinated cooling water and density of total viable count of marine bacteria and Manganese oxidizing bacteria on titanium surface exposed for various monthly durations to chlorinated cooling water.

Even the data stored in a single table of the warehouse may be from multiple sources. That is, the data represented in a table may be the consolidation of data from more than one published papers. In this case, credit has to be given to all the paper authors and organizations for the data.

By developing a material data warehouse, we aim to structure the available data in a consistent, accessible and logical form. MS SQL server is used to store the data. The data on various material properties received from different institutes has to be presented in a website. Even though the data is stored in a single warehouse, the display of data should satisfy all users who are interested in various aspects of the available data. So the data warehouse has to follow a modeling technique which can accommodate the multiple user views on material data.

\section{THE DATABASE MODELING PROCESS}

\subsection{An Overview of Dimensional Modeling}

Dimensional modeling always uses the concepts of facts (measures) and dimensions (context). Facts are normally quantitative measurements of the outcome of the business process being analyzed that can be aggregated and dimensions are groups of descriptors that define the facts. Dimensional modeling results in the generation of star schemas. Star schemas are constituted by a fact table in the centre surrounded by a range of dimensions. Dimension tables contain descriptive textual information with a single part primary key. The primary keys of dimension tables become foreign keys of the central fact table. These foreign keys constitute a multi-part primary key for that fact table, which, in turn, expresses a many-to-many relationship.

The fact table is the focus of dimensional analysis. It contains quantitative measurements that can be analyzed along different perspectives or dimensions, thus giving the data a multidimensional view. Each entry (row) in the fact table consists of a pointer (foreign key) to each of its adjoining dimensions and stores the data associated with those dimension table entries. The data represented by star-schemas 
are extensively denormalized with significant number of redundancies, thus improving analysis of data.

In addition to star schemas, dimensional models are also implemented using snowflake schemas. The snowflake schema is considered as an extension of the star schema [12]. It represents a dimensional model which is composed of a central fact table and a set of constituent dimension tables that are further broken up into sub-dimension tables. The snow flake schema attempts to reduce redundancies in star schema by introducing a degree of normalization into the dimensional model.

The star schema is considered to be the most efficient design suited for modeling data marts. The snowflake schema may suffer from potential performance issues from the relatively higher number of query joins needed as opposed to the star schema. But Martyn [11] explains that snowflake design can be used when a denormalized dimension table becomes very large and penalizes the star join operation, thus impeding the performance of the data warehouse. Also, the queries which will not access data from outer-level dimension tables can effectively execute against a star schema that contains smaller dimension tables. Therefore, under some circumstances, a snowflake schema is more efficient than a star schema [11].

\subsection{Identifying Facts and Dimensions: The Star Schema}

The objective of dimensional modeling is to represent heterogeneous data in a standard framework. Correctly identifying the dimensions and facts related to them is crucial in building a model that satisfies user's data requirements and analysis of data.

In a dimensional model, all dimensions can be thought of as symmetrically equal entry points into the fact table. The logical design of the dimensions is independent of the pattern of queries to which the database will be subjected to. In the case of material database, different users may be interested in different aspects of the data available in the database, such as, 1) The data available on a material type, 2) The data available on a property, 3) The source of data (i.e., data from a particular organization/ published in a particular journal etc...) or 4) The type of data (tables, graphs or microstructures). In accordance with this, the dimensions identified in the material database are materials, properties, test specification details and source of data.

The Materials dimension represents materials whose data are stored in the database. The attributes of this dimension are material class, material type and material subtype. The material id (MID) field acts as a primary key for the dimension.

The Property dimension stores the name, type and subtype of material properties whose data are available in the database. The property types currently available are mechanical, corrosion, non-destructive evaluation, thermal, optical, electrical and structural properties. Each property belongs to one of these types. The property name (PROPERTYNAME) attribute is a primary key for the dimension.

The Test Specifications dimension contains details of the experiments conducted to study a material's property. The data available on a material property will be complete only with an awareness of the test setup used by the researcher. The attributes of the test specifications dimension include chemical composition of the material, history of the material while testing, test standard followed by the researcher and conditions underwent by the material before the experiment (such as annealing, cold work etc...). The knowledge on the history of the material while testing and conditions under which tests were conducted are important for the user to make proper use of the data available as experiment results. For example, the database contains data regarding the sensitization property of 304 SS Austenitic Stainless Steel which is mill annealed and 15\% cold worked or creep fatigue property of $316 \mathrm{~L}(\mathrm{~N})$ Base Metal Austenitic Stainless steel which is solution annealed and quenched. The 'TESTID' attribute is the primary key for this dimension.

The Source dimension contains information on the organization which supplied the data, the publication in which the data appeared and authors of the data. So, the attributes of this dimension are organization, title and citation of the paper containing data, name of the publication and year of publication. 'SOURCEID' is the primary key for this dimension.

The fact table (Material_Facts) contains four foreign keys corresponding to the primary keys of each of the four dimensions. These foreign keys together define the multi-part/ compound primary key of the fact table. Unlike the common case, this fact table does not contain any quantitative measurements. Instead of numeric measurements, the table contains a field for the 'Type of data' available for a particular combination of dimension table entries. This attribute specifies whether the data available is in Tabular form (T) or Graphical form (G) or as Microstructure images (M) or a combination of these. The central fact table together with the dimension tables results in the star structure shown in Fig. 1. By virtue of this modeling, the user can retrieve data regarding the properties of a material under specific conditions or the material properties studied in a particular organization or information published in a journal of his choice etc...

\subsection{The Data Cube}

The fundamental idea of dimensional modeling is that data can be represented as a 'data cube', in which, the cells of the cube contain measured values and the edges define various dimensions of the data [16]. An OLAP cube is an abstract representation of a projection of an RDBMS relation. Given a relation of order $N$, consider a projection that subtends $X, Y$, and $Z$ as the key and $W$ as the residual attribute. Characterizing this as a function,

$W:(X, Y, Z) \rightarrow W$, 


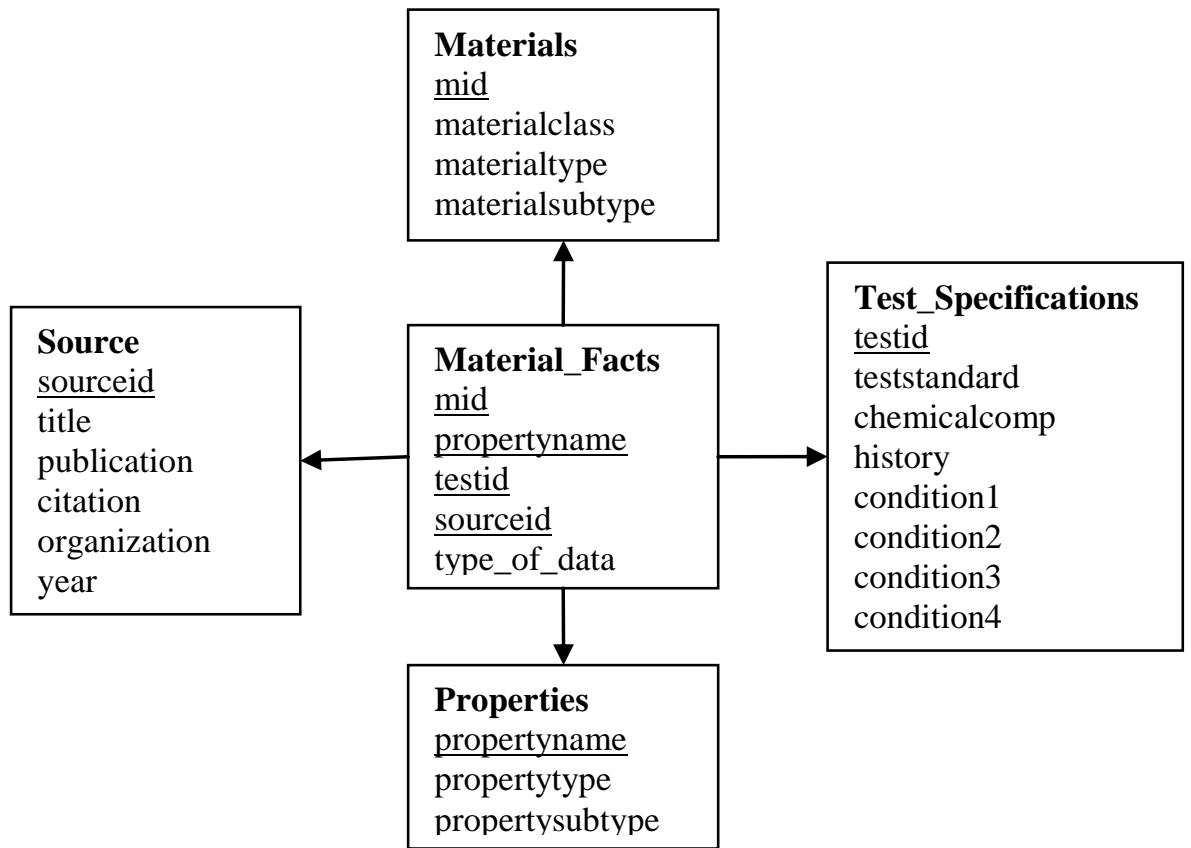

Fig 1: Star Schema

the attributes $X, Y$, and $Z$ correspond to the axes of the cube, while the $W$ value into which each $(X, Y, Z)$ triple maps corresponds to the data element that populates each cell of the cube. Although referred to as 'cube', an OLAP cube can be two dimensional, three dimensional or higher dimensional (hyper-cube).The most common operations on an OLAP cube are slice-and-dice to define a sub-cube, drill-down and roll-up to perform aggregation and disaggregation respectively along a hierarchical dimension, drill-across to combine multiple cubes, ranking to find the outlier values, and rotating to see the data grouped by other dimensions. The attributes of any dimensions of the cube can be organized based on parent- child relationships, thus resulting in a hierarchy. The materials data cube as well as the hierarchical relationships among the attributes of the dimensions is represented in Fig. 2 .

\subsection{Snowflake Schema}

As explained earlier, the data available on each material property is in the form of one or more tables. This data cannot be directly fit into the star structure. To incorporate data available on material properties, the star schema is extended to a snowflake schema. In snowflake schemas, one or more of the dimension tables are normalized into sub-dimensions.

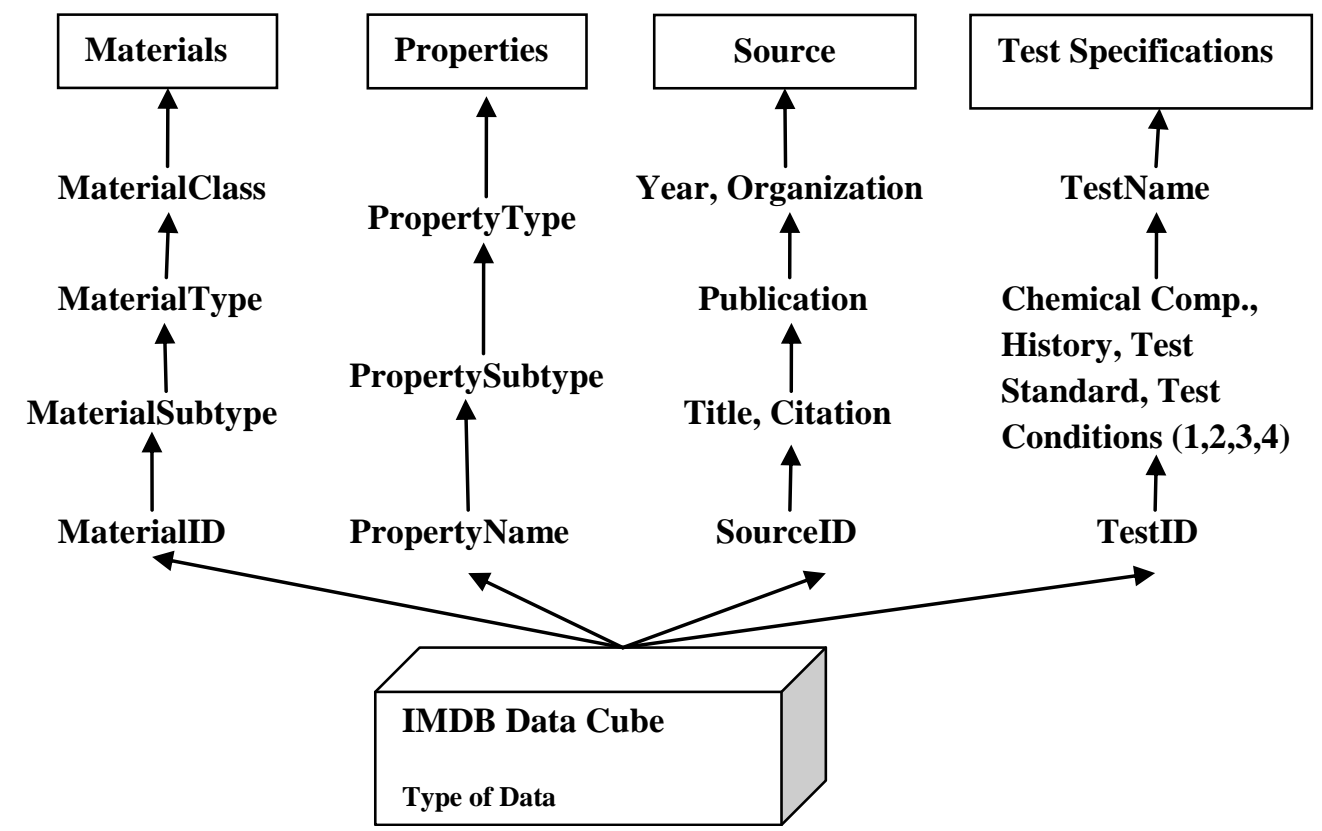

Fig 2: Data Cube with hierarchical relationships in Dimensions

The 'Test Specifications' dimension holds the test details for each material corresponding to different tests. The material property data obtained as experiment results are stored as data tables which form sub-dimensions of the
'Test Specifications' dimension. The primary key 'TESTID' of this dimension acts as a foreign key for the data tables.

As said earlier, apart from tables, the data may be in the form of graphs or microstructure images. Currently, the images for 
graphs and microstructures are not stored inside the database considering space requirements. Instead, the details of the graph and microstructure data along with their location in the computer hard disk are stored as separate 'Graph' and 'Microstructure' tables in the database. These tables also form sub-dimensions of the 'Test_Specifications' dimension with 'TESTID' as a foreign key.

The snowflake design with data tables of sensitization, biofouling and hardness properties together with graph and microstructure tables is given in Fig. 3 .

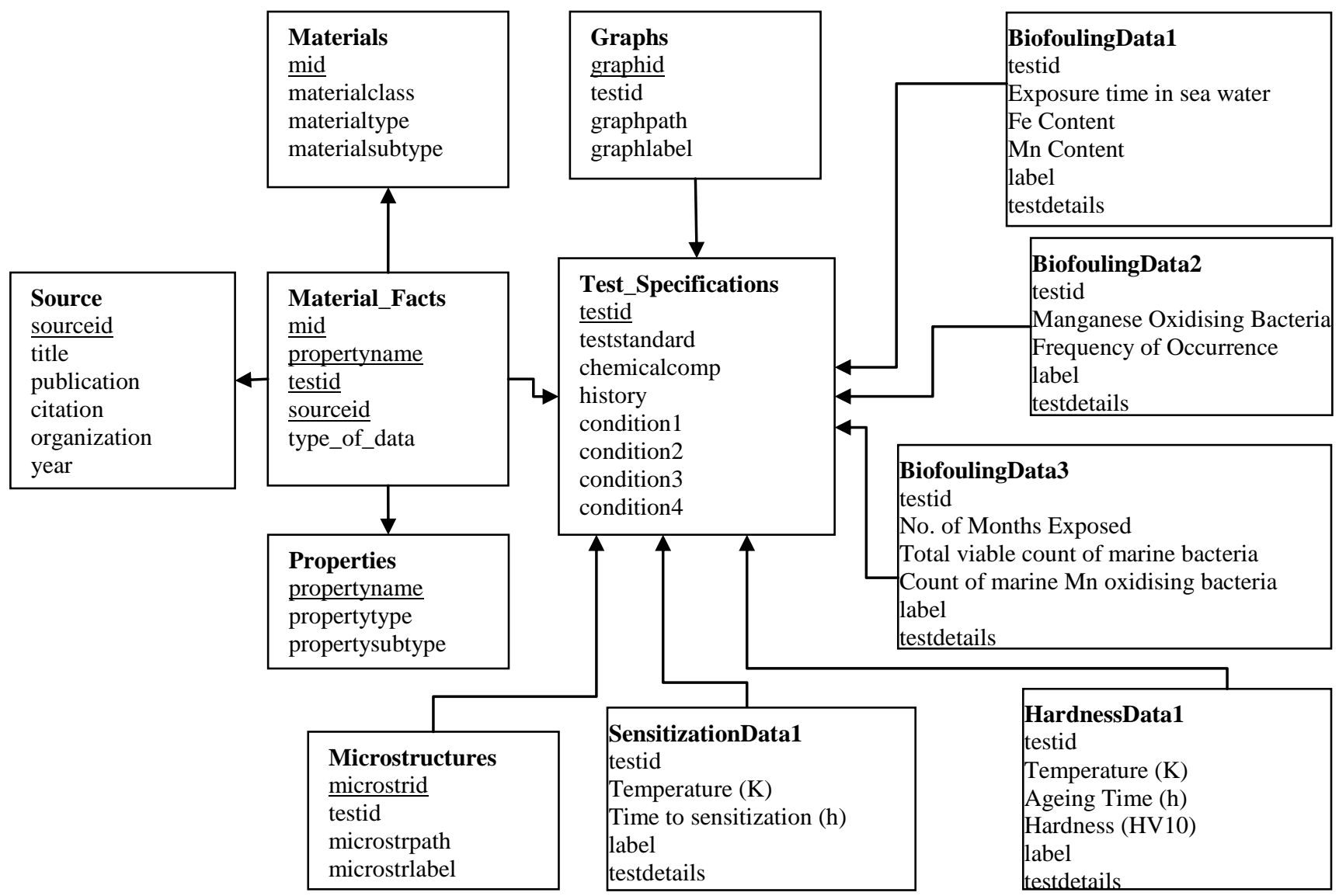

Fig 3: Snowflake Schema

\section{RESULTS AND DISCUSSION}

As said earlier, the dimensional data is represented as a data cube which supports many operations including slicing of the cube. A slice is a subset of a multi-dimensional cube corresponding to a single value for one or more members of the dimensions not in the subset. From an end user perspective, a slice refers to a two- dimensional page selected from the cube. In Fig. 4, Fig. 5, Fig. 6 and Fig. 7, slices of the material data cube formed by selecting one member from a particular dimension are shown.

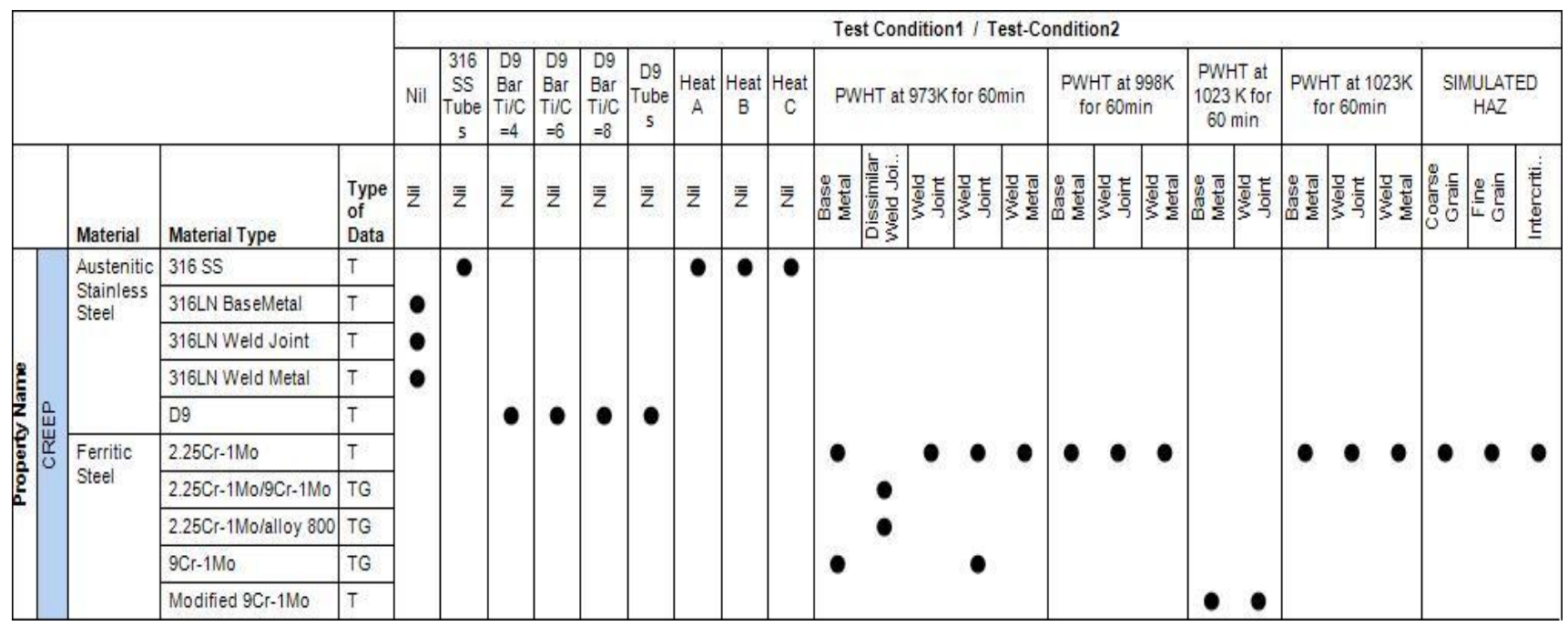

Fig 4: Data Sub-Cube for PropertyName = 'CREEP' 


\begin{tabular}{|c|c|c|c|c|c|c|c|c|}
\hline & & & & & \multicolumn{4}{|c|}{ Property Type / Property Name } \\
\hline & & & & & \multicolumn{3}{|c|}{ CORROSION } & MECHANICAL \\
\hline & Material & Material Type & Year & $\begin{array}{l}\text { Type } \\
\text { of } \\
\text { Data }\end{array}$ & $\begin{array}{l}\text { HYDROGEN } \\
\text { TRANSPORT }\end{array}$ & SENSITIZATION & $\begin{array}{c}\text { SODIUM } \\
\text { CORROSION }\end{array}$ & CREEP \\
\hline \multirow{10}{*}{ 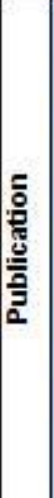 } & \multirow{8}{*}{\begin{tabular}{|l} 
Austenitic \\
Stainless \\
Steel
\end{tabular}} & 304 SS & 1989 & T & \multicolumn{3}{|c|}{ ? } & \\
\hline & & $316 \mathrm{SS}$ & 1989 & $T$ & \multicolumn{3}{|c|}{ - } & \\
\hline & & 316(L) Weld Metal & 2006 & $T$ & \multicolumn{3}{|c|}{ ? } & \\
\hline & & $316(\mathrm{~N})$ Weld Metal & 2006 & $T$ & \multicolumn{3}{|c|}{ - } & \\
\hline & & $316 \mathrm{~L}(\mathrm{~N})$ & 2002 & $T$ & \multicolumn{3}{|c|}{ - } & \\
\hline & & $316 \mathrm{~L}(\mathrm{~N}) 2$ & 1994 & T & \multicolumn{3}{|c|}{ - } & \\
\hline & & \multirow[t]{2}{*}{ 316LN BaseMetal } & 1999 & $T$ & & & & - \\
\hline & & & 2008 & T & \multicolumn{3}{|r|}{ - } & \\
\hline & \multirow{2}{*}{\begin{tabular}{|l} 
Ferritic \\
Steel
\end{tabular}} & $2.25 \mathrm{Cr}-1 \mathrm{Mo}$ & 2001 & $T$ & \multicolumn{3}{|l|}{ ○ } & \\
\hline & & Modified $9 \mathrm{Cr}-1$ Mo & 1999 & $T$ & \multicolumn{3}{|l|}{ ○ } & \\
\hline
\end{tabular}

Fig 5: Data Sub-Cube for Publication = 'Journal of Nuclear Materials'

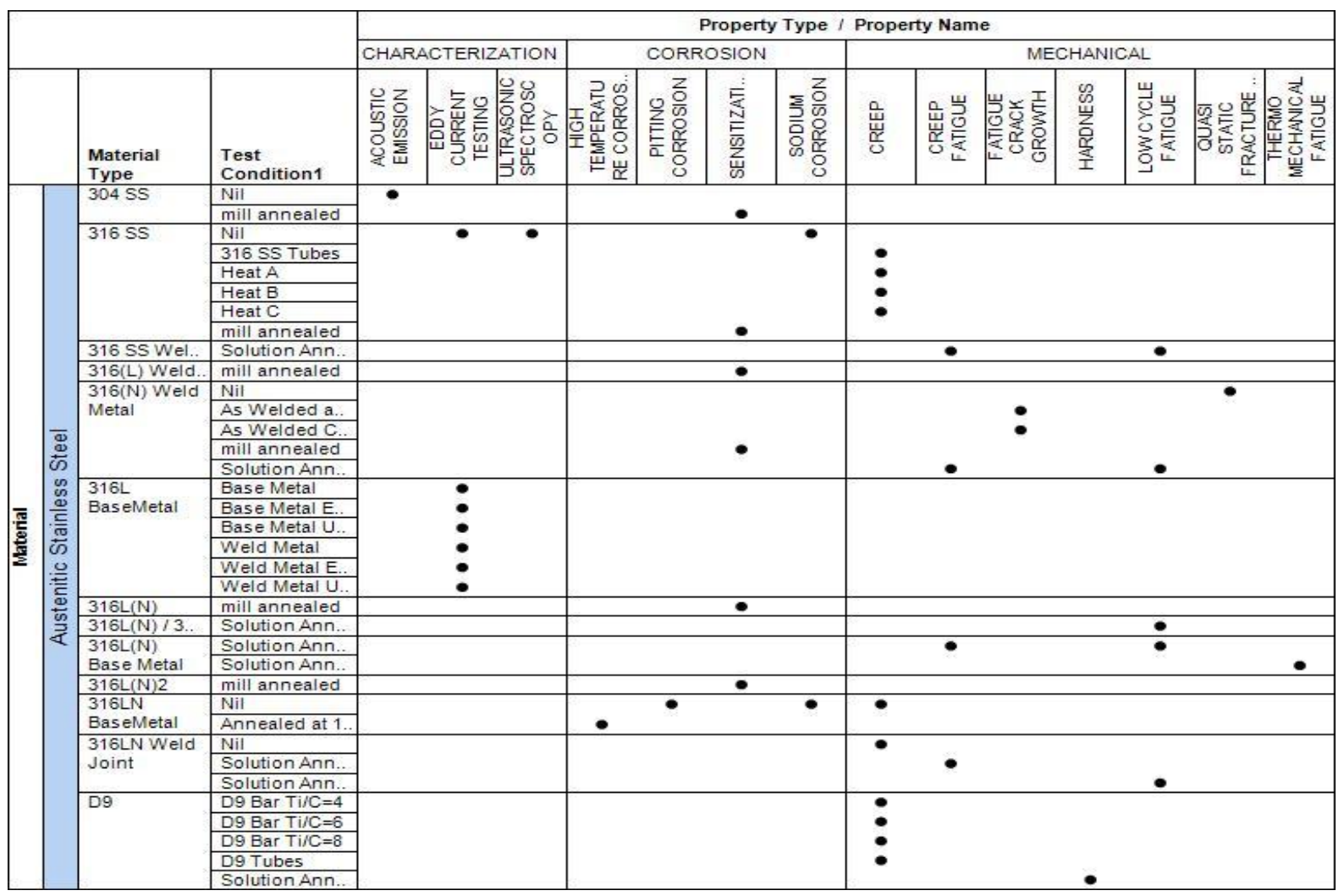

Fig 6: Data Sub-Cube for Material Class = 'Austenitic Stainless Steel' 


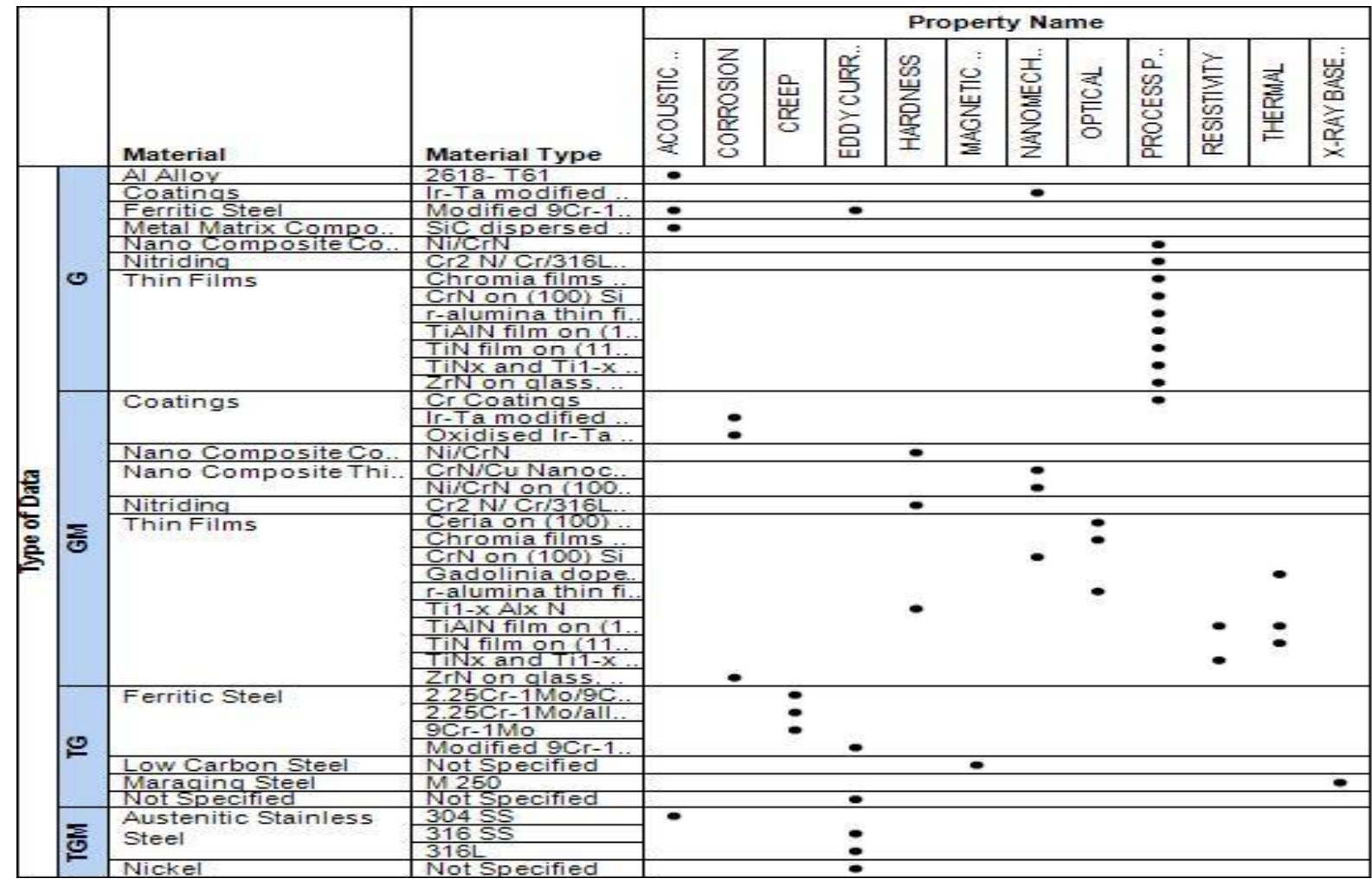

Fig 7: Data Sub-Cube for Materials and Properties for which Graph data is available i.e., typeofdata in ('G','GM','TG','TGM')

\section{CONCLUSION}

This paper describes the development of a material database which brings together the materials data generated in different research institutes across the country using dimensional modeling techniques. The dimensional model forms a standard, predictable framework for representing the data and enhancing the user's understandability of data. The star schema generated using the dimensional model is highly flexible and extensible to accommodate new data elements and design decisions. This is highly useful in the case of material database since it is a dynamic data store that keeps on growing by accepting more and more data on diverse material properties from participating organizations. Fresh data can be included in the database by simply adding new data rows to the fact and dimension tables.

In the dimensional model, each dimension is treated as a separate entry point to the fact table. Every dimension is equivalent. This feature makes the user interfaces and query strategies symmetrical.

The IMDB website is designed as a fully data driven website independent of the addition or modification of material property data. The snowflake design which treats different data tables as sub dimensions of the 'Test_Specifications' dimension helps the application to identify data corresponding to a particular material and a property, thus eliminating the need for hard coding the data table name in the application. The same case works with graph and microstructure data as well. Thus, the existing application can continue to work in spite of changes in database.

\section{ACKNOWLEDGMENTS}

We express our sincere gratitude to Dr. U. Kamachi Mudali, Dr. M. Valsan and Mr. R.V. Subba Rao working at Indira Gandhi Centre for Atomic Research for their valuable guidance and contributions. We are extremely thankful to Dr. Baldev Raj, our former director and Mr. S.C. Chetal, our current director for their support and encouragement.

\section{REFERENCES}

[1] S. Rajeswari, S.A.V. Satya Murty, Baldev Raj et al. (2010), Challenges in designing a database for corrosion and mechanical properties of materials, In Proceedings of the Second Asian Symposium for Materials Database, Sanya, China. pp. 339-344.

[2] W.H. Inmon (1993), Building the Data Warehouse, Wiley.

[3] R. Kimball (1997), A Dimensional Modeling Manifesto http://ralph12.securesites.net/html/articles_search/articles 1997/9708d15.html Accessed 23 Dec 2010.

[4] R. Kimball, M. Ross, W. Thornthwaite, and L. Reeves. (1998), The Data Warehouse Lifecycle Toolkit: Expert Methods for Designing, Developing, and Deploying Data Warehouses, Wiley.

[5] R. Kimball, M. Ross, W. Thornthwaite, J. Mundy and B. Becker. (1998), The Data Warehouse Lifecycle Toolkit, Wiley.

[6] M.A. Kortini and L.D. Moody (2000), From Enterprise Models to Dimensional Models: A Methodology for Data Warehouses and Data Mart Design, Proceedings of 
the International Workshop on Design and Management of Data Warehouses, Stockholm, Sweden. 5.1-5.12.

[7] M. Golfarelli, D. Maio, and S. Rizzi. (1998), The dimensional fact model: a conceptual model for data warehouses, International Journal of Cooperative Information Systems, Volume 7. pp. 215-247.

[8] M. Golfarelli, D. Maio, and S. Rizzi (1998), Conceptual Design of Data Warehouses from E/R Schema, In Proceedings of the Thirty-First Annual Hawaii international Conference on System Sciences, Volume 7. pp.334.

[9] I.Y. Song, W. Rowen, C. Medsker and E. Ewen. (2001), An Analysis of Many-to-Many Relationships Between Fact and Dimension Tables in Dimension Modeling, Proceedings of the International Workshop on Design and Management of Data Warehouses, Interlaken, Switzerland. pp. 6.1-6.13.

[10] I.Y. Song and M.E. Jones (2008), Dimensional modeling: Identification, classification and evaluation of patterns, Decision Support Systems, Volume 45, Issue 1. pp. 59-76.

[11] T. Martyn (2004), Reconsidering Multi-Dimensional Schemas, ACMs Special Interest Group on Management of Data, Volume 33, Issue 1. pp. 83-88.
[12] M. Levene and G. Loizou (2003), Why is the Snowflake Schema a good data warehouse design, Information Systems, Volume 28, Issue 3. pp. 225-240.

[13] Xiu-Juan Zhang, Ke-Zhang Chen, Xin-An Feng (2008), Material database for the material design of components made of a multiphase perfect material, Materials \& Design, Volume 29, Issue 6. pp. 1131-1144

[14] P.S. Ramalhete, A.M.R. Senos, C. Aguiar, Digital tools for material selection in product design, Materials \& Design, Volume 31, Issue 5, May 2010. pp. 2275-2287

[15] P.J. Karditsas, G. Lloyd, M. Walters, A. Peacock (2006), The European Fusion Material properties database, Fusion Engineering and Design, Volume 81, Issues 8-14. pp. 1225-1229

[16] Vassiliadis P (1998), Modeling Multidimensional Databases, Cubes and Cube Operations, In Proceedings of the 10th International Conference on Scientific and Statistical Database Management (SSDBM '98), IEEE Computer Society. pp. 53-62. 